\section{Comparing the neurologic outcomes of patients with out-of-hospital cardiac arrest according to prehospital advanced airway management method and transport time interval}

\author{
Sol Kim', Dong Eun Lee ${ }^{1 *}$, Sungbae Moon', Jae Yun Ahn', Won Kee Lee², \\ Jong Kun Kim', Jungbae Park', Hyun Wook Ryoo ${ }^{1 *}$ \\ 'Department of Emergency Medicine, Kyungpook National University School of Medicine, Daegu, Korea \\ ${ }^{2}$ Medical Research Collaboration Center in Kyungpook National University Hospital, Kyungpook National \\ University School of Medicine, Daegu, Korea
}

Objective The incidences of prehospital advanced airway management by emergency medical technicians in South Korea are increasing; however, whether this procedure improves the survival outcomes of patients experiencing out-of-hospital cardiac arrest remains unclear. The present study aimed to investigate the association between prehospital advanced airway management and neurologic outcomes according to a transport time interval (TI) using the Korean Cardiac Arrest Research Consortium database.

Methods We retrospectively analyzed the favorable database entries that were prospectively collected between October 2015 and December 2016. Patients aged 18 years or older who experienced cardiac arrest that was presumed to be of a medical etiology and that occurred prior to the arrival of emergency medical service personnel were included. The exposure variable was the type of prehospital airway management provided by emergency medical technicians. The primary endpoint was a favorable neurologic outcome.

Results Of 1,871 patients who experienced out-of-hospital cardiac arrest, 785 (42.0\%), 121 (6.5\%), and 965 (51.6\%) were managed with bag-valve-mask ventilation, endotracheal intubation (ETI), and supraglottic airway (SGA) devices, respectively. SGAs and ETI provided no advantage in terms of favorable neurologic outcome in patients with $\Pi \mathrm{Tls} \geq 12$ minutes (odds ratio [OR], 1.37; confidence interval [Cl], 0.65-2.87 for SGAs; $\mathrm{OR}, 1.31$; $\mathrm{Cl}, 0.30-5.81$ for ETI) or in patients with $\mathrm{Tl}<12$ minutes (OR, 0.57; $\mathrm{Cl}, 0.31-1.07$ for SGAs; $\mathrm{OR}, 0.63 ; \mathrm{Cl}, 0.12-3.26$ for $\mathrm{ETI})$.

Conclusion Neither the prehospital use of SGA nor administration of ETI was associated with superior neurologic outcomes compared with bag-valve-mask ventilation.

Keywords Airway management; Emergency medical services; Intubation, intratracheal; Out-ofhospital cardiac arrest
eISSN: 2383-4625

Received: 7 January 2019

Revised: 9 April 2019

Accepted: 18 April 2019

${ }^{*}$ Correspondence to: Dong Eun Lee Department of Emergency Medicine, School of Medicine, Kyungpook National University, 680 Gukchaebosang-ro, Jung-gu, Daegu 41944, Korea

E-mails: delee@knu.ac.kr ORCID

http://orcid.org/0000-0002-2057-5261

*Correspondence to: Hyun Wook Ryoo Department of Emergency Medicine, School of Medicine, Kyungpook National University, 680 Gukchaebosang-ro, Jung-gu, Daegu 41944, Korea E-mail ryoo@knu.ac.kr ORCID http://orcid.org/0000-0002-1361-9887

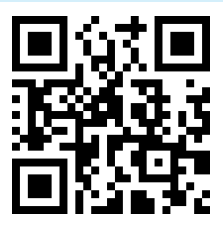

How to cite this article:

Kim S, Lee DE, Moon S, Ahn JY, Lee WK, Kim JK, Park J, Ryoo HW. Comparing the neurologic outcomes of patients with outof-hospital cardiac arrest according to prehospital advanced airway management method and transport time interval. Clin Exp Emerg Med 2020;7(1):21-29.

This is an Open Access article distributed under the terms of the Creative Commons Attribution Non-Commercial License (http:// creativecommons.org/licenses/by-nc/4.0/). 


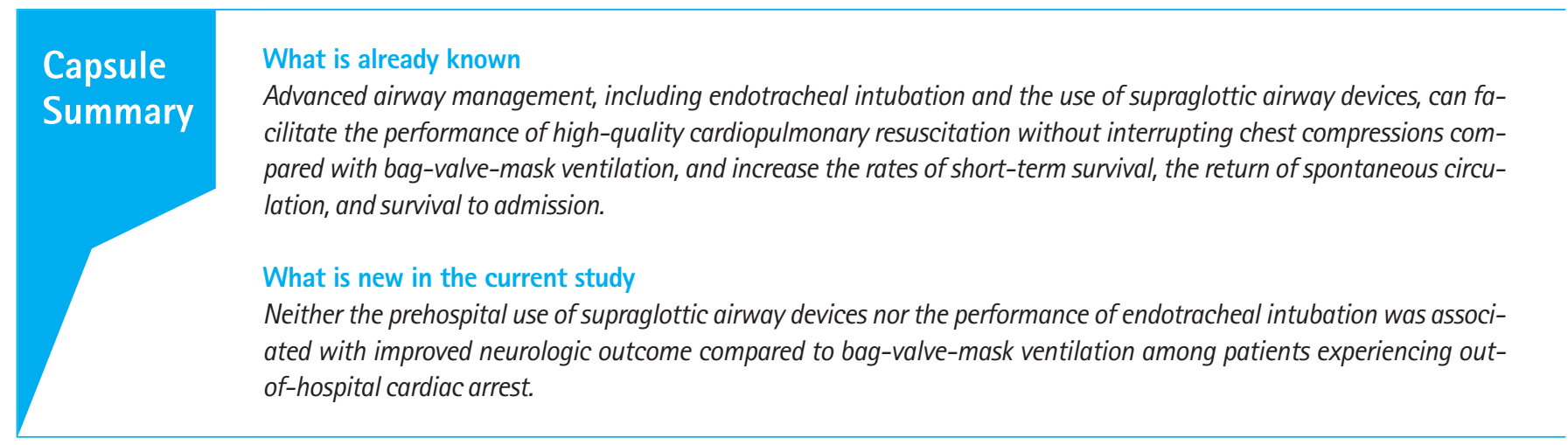

\section{INTRODUCTION}

During cardiopulmonary resuscitation (CPR) on a patient experiencing out-of-hospital cardiac arrest (OHCA), it is recommended that healthcare providers maintain bag-valve-mask (BVM) ventilation before performing advanced airway management. ${ }^{1}$ BVM ventilation has the disadvantage of producing complications such as aspiration and pneumonia due to gastric expansion or regurgitation. ${ }^{2,3}$ After the return of spontaneous circulation (ROSC), performing endotracheal intubation (ETI) elicits controlled and effective ventilation that is necessary while transporting the patient with OHCA to the emergency department. ${ }^{4}$ Advanced airway management including ETI or the use of supraglottic airway (SGA) devices can facilitate the performance of high-quality CPR without interrupting chest compressions (as would be required with BVM ventilation) and increases the rates of short-term survival, ROSC, and survival to admission. ${ }^{5,6}$ ETI, which prevents airway obstruction and facilitates airway management, is preferred by hospitals, although performing it requires high skill. ${ }^{7}$ Various SGAs such as the esophageal obturator airway, laryngeal mask, laryngeal tube, and I-gel device are being disseminated to assist healthcare providers to maintain open airways with minimal training. ${ }^{8,9}$

With the increase in the proportion of level 1 emergency medical technicians (EMTs) among the 119 emergency medical service (EMS) agencies in South Korea, there has been an increasing trend toward performing advanced airway management, including ETI and SGA use, during the prehospital phase. However, the effects of prehospital airway management on the neurologic outcomes of patients experiencing OHCA remain unclear despite previous investigations. $^{3,10-12}$ Previous studies showed that the direct transfer of OHCA patients to a percutaneous coronary intervention-capable center or a critical care center improved survival outcomes; as a result, strategies aimed at the regionalization of postresuscitation care for OHCA patients are emerging. ${ }^{13-15}$ A previous meta- analysis revealed that the transport time interval (TI) was not associated with neurologic outcomes or survival to discharge among OHCA patients. ${ }^{16}$ Advanced airway management is associated with an improved no-flow ratio when performing $\mathrm{CPR}^{6}$ and can reduce complications commonly associated with BVM ventilation such as pneumonia, gastric expansion, and regurgitation. ${ }^{2,3}$ Therefore, it is possible that prehospital advanced airway management may improve the outcomes of patients requiring extended transport times. Nevertheless, the association between prehospital airway management and survival outcomes according to TTI remains unclear. Additionally, there have been no studies in South Korea on the effects of prehospital airway management and ventilation methods on survival rates and neurologic outcomes according to $\mathrm{TI}$ in patients experiencing OHCA.

Considering that prehospital airway management can minimize chest compression interruptions, provide effective ventilation, and reduce airway complications in patients with $\mathrm{OHCA}_{1}^{2,3,6}$ we hypothesized that prehospital advanced airway management will have advantages over BVM ventilation with respect to neurologic outcomes, and that the beneficial effect of advanced airway management will increase with the length of the TI. The present study aimed to investigate the association between the prehospital airway management method (BVM, ETI, or SGA) and neurologic outcomes in patients with OHCA according to TI using data from the Korean Cardiac Arrest Research Consortium (KoCARC) database. ${ }^{17}$

\section{METHODS}

\section{Study design, setting, and subjects}

We retrospectively analyzed data from the KoCARC database that was recorded between October 2015 and December 2016. Patients aged 18 years or older who experienced cardiac arrest that was presumed to be of a medical etiology and that occurred prior to 
the arrival of EMS personnel on the scene were included in this study. The KoCARC registry excluded OHCA patients with terminal illnesses documented in their medical records as well as those under hospice care, those who were pregnant, and those with "do not resuscitate" directives. Furthermore, we also excluded the following groups: patients with OHCA verifiably caused by nonmedical etiologies such as trauma, drowning, poisoning, burn, asphyx$i a$, and hanging ${ }^{17}$; patients who transferred from other hospitals and had insufficient prehospital time variables and/or unclear prehospital airway management methods; and patients who experienced cardiac arrest during transport to the hospital (whether in an ambulance or otherwise). In South Korea, if three or more EMTs are dispatched to the location of a patient with cardiac arrest, they are required to perform ETI or use SGAs while administering effective chest compressions in the field according to field treatment guidelines. This study was approved by the institutional review board of Kyungpook National University Hospital (201511-013-007). The requirement for informed consent was waived owing to the retrospective nature of this study.

\section{Data source}

The KoCARC registry is a multicenter nationwide network of 64 participating institutions for data collection and collaborative research from across South Korea. Data are collected according to standardized Utstein-style templates for OHCA to facilitate uniform reporting using precisely defined variables and outcomes. Data are error-checked prior to consolidation with the master dataset. The quality management committee provides feedback regarding quality management processes to the research coordinators and investigators. ${ }^{17}$

\section{Outcomes and variables}

The exposure variable in this study was the type of prehospital airway management provided by EMTs. The subjects were divided into three groups according to the prehospital airway management method (BVM, ETI, or SGA); patient demographics, arrest characteristics, and survival outcomes were compared among the three groups.

Neurologic status was assessed using cerebral performance category scores, which are based on a five-point scale in which scores of 1 (good recovery) and 2 (moderate disability) indicated favorable neurologic outcome. The primary endpoint was a favorable neurologic outcome, whereas the secondary endpoint was survival to hospital discharge. We used the following a favorable registry core variables: (1) patient demographics (i.e., sex, age, medical history of hypertension, diabetes, and dyslipidemia); (2) community resuscitation (i.e., 'witnessed' status, bystander CPR, and location of cardiac arrest occurrence); (3) EMS resuscitation (i.e., initial electrocardiogram rhythm, prehospital defibrillation, prehospital epinephrine administration, prehospital ROSC, elapsed time from the initial call to ambulance arrival at the scene [response time interval], elapsed time from arrival at the scene to departure [scene time interval], and elapsed time from departure to arrival at the emergency department [TII]); and (4) hospital resuscitation and postresuscitation care (i.e., performed extracorporeal membrane oxygenation, coronary angiography, and target temperature management). ${ }^{17}$

\section{Statistical analysis}

The significance of differences between groups was tested using analysis of variance with post-hoc analysis (with multiple com-

KoCARC database between

October 1, 2015 and December 31, 2016

3,187 Out-of-hospital cardiac arrest

94 Under 18 years with cardiac arrest

3,093 Adult OHCA, KoCARC registry

231 Transferred from other hospitals

912 Unknown EMS time status

9 Unknown prehospital airway management

1,871 Adult OHCA, KoCARC registry

70 Cardiac arrest occurred in ambulance

\section{Bag-valve-mask}

965 Supraglottic airway
121 Endotracheal intubation

Fig. 1. Patients with out-of-hospital cardiac arrest analyzed in the current study. KoCARC, Korean Cardiac Arrest Research Consortium; OHCA, out-ofhospital cardiac arrest; EMS, emergency medical service. 
parisons using the Bonferroni method) for continuous variables and Pearson chi-square test for categorical variables. The association between prehospital advanced airway management and outcomes was assessed using logistic regression analysis according to the $\Pi \mathrm{I}$ after classifying the patients into two groups, $\Pi \mathrm{II} \geq 12$ vs. $<12$ minutes; this cutoff was the time point found to show the greatest difference in the direction of this association. To assess homogeneity across the two groups, the Breslow-Day test was performed to determine OHCA outcomes by 12 minutes of transport time. Our subgroup analysis revealed that a TI of 12 minutes presented the least homogeneity on Breslow-Day testing $(P=0.028$ for neurologic outcome, $P=0.040$ for survival to discharge). Logistic regression analysis was performed by adjusting the confounding variables based on a TTI of $\geq 12$ and of $<12$ minutes to examine the association between prehospital advanced airway management and outcomes. We included potential confounding variables such as sex, age, response time interval, scene time interval, location of cardiac arrest occurrence, shockable

Table 1. Baseline characteristics and outcomes of the study population based on the prehospital airway management method

\begin{tabular}{|c|c|c|c|c|c|}
\hline & \multirow{2}{*}{ Total $(n=1,871)$} & \multicolumn{3}{|c|}{ Prehospital airway management } & \multirow{2}{*}{ P-value } \\
\hline & & BVM $(n=785)$ & SGA $(n=965)$ & $\operatorname{ETI}(n=121)$ & \\
\hline Sex & & & & & $<0.001$ \\
\hline Male & $1,223(65.4)$ & $479(61.0)$ & $673(69.7)$ & $71(58.7)$ & \\
\hline Female & $648(34.6)$ & 306 (39.0) & $292(30.3)$ & $50(41.3)$ & \\
\hline Age $(y r)$ & & & & & 0.003 \\
\hline$<65$ & $758(40.5)$ & $335(42.7)$ & $391(40.5)$ & $32(26.4)$ & \\
\hline$\geq 65$ & $1,113(59.5)$ & $450(57.3)$ & $574(59.5)$ & 89 (73.6) & \\
\hline Age (yr) & $70(56-78)$ & $68(55-78)$ & $70(56-78)$ & $74(63-81)$ & $0.026^{\mathrm{al}}$ \\
\hline RTI (min) & $7(6-10)$ & $7(5-10)$ & $8(6-10)$ & $7(5-10)$ & 0.655 \\
\hline STI (min) & $11(8-17)$ & $9(6-13)$ & $14(10-20)$ & $14(10-20)$ & $<0.001^{b)}$ \\
\hline TI (min) & $10(6-14)$ & $10(7-14)$ & $9(6-13)$ & $10(8-15)$ & $0.007^{c)}$ \\
\hline Location & & & & & $<0.001$ \\
\hline Home & $1,261(67.4)$ & $478(60.9)$ & $689(71.4)$ & $94(77.7)$ & \\
\hline Public place & $419(22.4)$ & $218(27.8)$ & $186(19.3)$ & $15(12.4)$ & \\
\hline Other/unknown & $191(10.2)$ & $89(11.3)$ & $90(9.3)$ & $12(9.9)$ & \\
\hline \multicolumn{6}{|l|}{ Comorbidities } \\
\hline Hypertension & $768(41.0)$ & $302(38.5)$ & $406(42.1)$ & $60(49.6)$ & 0.045 \\
\hline Diabetes mellitus & $482(25.8)$ & $178(22.7)$ & $261(27.0)$ & $43(35.5)$ & 0.005 \\
\hline Hyperlipidemia & $96(5.1)$ & $48(6.1)$ & $39(4.0)$ & $9(7.4)$ & 0.073 \\
\hline Witnessed cardiac arrest & $1,112(59.2)$ & $502(64.0)$ & $536(55.5)$ & 70 (57.9) & 0.002 \\
\hline Bystander CPR & $961(51.4)$ & $375(47.8)$ & $524(54.3)$ & $62(51.2)$ & 0.025 \\
\hline Initial shockable rhythm & $366(19.6)$ & $138(17.6)$ & $207(21.5)$ & $21(17.4)$ & 0.104 \\
\hline Prehospital defibrillation & $483(25.8)$ & $185(23.6)$ & $271(28.1)$ & $27(22.3)$ & 0.066 \\
\hline Prehospital epinephrine administration & $293(15.7)$ & $38(4.8)$ & $226(23.4)$ & $29(24.0)$ & $<0.001$ \\
\hline Prehospital ROSC & $242(12.9)$ & $91(11.6)$ & $134(13.9)$ & $17(14.0)$ & 0.339 \\
\hline \multicolumn{6}{|l|}{ Hospital treatment } \\
\hline CAG & $204(10.9)$ & $80(10.2)$ & $108(11.2)$ & $16(13.2)$ & 0.559 \\
\hline ТाM & $143(7.6)$ & $56(7.1)$ & $73(7.6)$ & $14(11.6)$ & 0.230 \\
\hline ECMO & $46(2.5)$ & $19(2.4)$ & $19(2.0)$ & $8(6.6)$ & 0.008 \\
\hline Survival to discharge & & & & & 0.130 \\
\hline Yes & $192(10.3)$ & $93(11.8)$ & $87(8.9)$ & $13(10.7)$ & \\
\hline No & $1,679(89.7)$ & $692(88.2)$ & $888(91.1)$ & $109(89.3)$ & \\
\hline Good neurologic outcome & & & & & 0.044 \\
\hline Yes & $130(6.9)$ & $68(8.7)$ & $56(5.8)$ & $6(5.0)$ & \\
\hline No & 1,741 (93.1) & 717 (91.3) & 909 (94.2) & 115 (95.0) & \\
\hline
\end{tabular}

Values are presented as number (\%) or median (interquartile range).

BVM, bag-valve-mask; SGA, supraglottic airway; ETI, endotracheal intubation; RTI, response time interval; STI, scene time interval; TTI, transport time interval; CPR, cardiopulmonary resuscitation; ROSC, return of spontaneous circulation; $C A G$, coronary angiography; $\Pi M$, targeted temperature management; ECMO, extracorporeal membrane oxygenation.

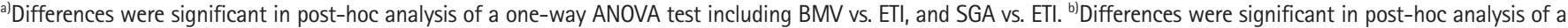
one-way ANOVA test including BMV vs. SGA, and BMV vs. ETI. 'Differences were significant in post-hoc analysis of a one-way ANOVA test including BMV vs. SGA. 
rhythm, 'witnessed' status, bystander CPR, prehospital defibrillation, and prehospital epinephrine administration. The results are expressed as odds ratios (ORs) and 95\% confidence intervals (Cls). All statistical tests were performed using SAS software ver. 9.4 (SAS Institute Inc., Cary, NC, USA), and P-values $<0.05$ were considered statistically significant.

\section{RESULTS}

\section{Baseline characteristics and outcomes of patients} according to prehospital airway management method Among the 3,187 OHCA subjects registered in the KoCARC database between October 1, 2015 and December 31, 2016, 94 patients aged $<18$ years were excluded. Additionally, 231 patients who were transferred from a different hospital prior to admission to the participating hospital, 912 who had unclear or unknown time variables between prehospital EMS site arrival and hospital arrival, 9 whose prehospital airway management methods were unknown, and 70 who experienced cardiac arrest in the ambulance were excluded. Ultimately, 1,871 patients were included in the final analysis. The subjects were divided into the following three groups according to the prehospital airway management method: BVM $(n=785)$, SGA $(n=965)$, and ETI $(n=121)$ (Fig. 1 and Table 1).
Patients in the ETI group were the oldest among the three, with subjects older than 65 years of age (median, 74 years) accounting for $73.6 \%$ (89 subjects) of the entire group. The BVM group had the lowest median on-scene time at 9 minutes, the highest rate of cardiac arrest occurring in a public place at $27.8 \%$ (218 subjects), and the highest witnessed cardiac arrest rate at 64\% (502 subjects). Moreover, 23.4\% and 24\% of subjects in the SGA and ETI groups, respectively, required prehospital epinephrine administration; these rates were higher than that for the BVM group. Among the three groups, the BVM group had the highest survival to discharge rate and the highest proportion of patients with good neurologic outcomes at $11.8 \%$ and $8.7 \%$, respectively.

\section{Analysis of the association between prehospital airway management and outcomes}

After adjustment for potential confounders, neither SGA nor ETI use was associated with better neurologic outcomes than BVM ventilation $(\mathrm{OR}, 0.59 ; \mathrm{Cl}, 0.33-1.06$ for $\mathrm{SGA} ; \mathrm{OR}, 0.77 ; \mathrm{Cl}, 0.21-$ 2.83 for ETI). Similarly, the use of SGA or ETI was not associated with improved survival to discharge compared to BVM ventilation (OR, 0.88; Cl, 0.57-1.38 for SGA; OR, 1.52; Cl, 0.66-3.47 for ETI) (Table 2).

Table 2. Effect of prehospital advanced airway management on survival outcomes

\begin{tabular}{|c|c|c|c|c|}
\hline \multirow{2}{*}{ Prehospital airway management } & \multicolumn{2}{|c|}{ Good neurologic outcome } & \multicolumn{2}{|c|}{ Survival to discharge } \\
\hline & Crude OR $(95 \% \mathrm{Cl})$ & Adjusted $\mathrm{OR}^{\mathrm{a}}(95 \% \mathrm{Cl})$ & Crude OR $(95 \% \mathrm{Cl})$ & Adjusted $\mathrm{OR}^{\mathrm{a})}(95 \% \mathrm{Cl})$ \\
\hline BVM & Reference & Reference & Reference & Reference \\
\hline SGA & $0.65(0.45-0.94)$ & $0.59(0.33-1.06)$ & $0.73(0.53-0.99)$ & $0.88(0.57-1.38)$ \\
\hline ETI & $0.55(0.23-1.30)$ & $0.77(0.21-2.83)$ & $0.90(0.48-1.66)$ & $1.52(0.66-3.47)$ \\
\hline
\end{tabular}

$\mathrm{OR}$, odds ratio; $\mathrm{Cl}$, confidence interval; $\mathrm{BVM}$, bag-valve-mask; SGA, supraglottic airway; $\mathrm{ETI}$, endotracheal intubation.

${ }^{\text {a) }}$ djusted for sex, age, response time interval, scene time interval, transport time interval, location of cardiac arrest occurrence, shockable rhythm, witnessed status, bystander cardiopulmonary resuscitation, prehospital defibrillation, and prehospital epinephrine administration.
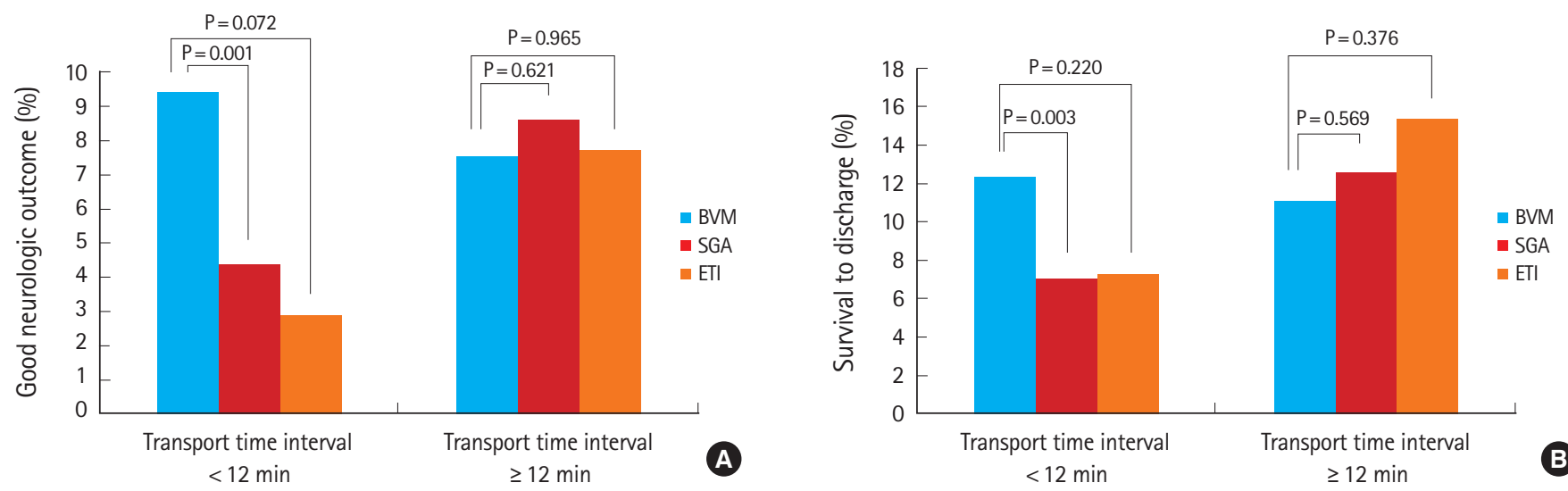

Fig. 2. Rates of good neurologic outcome (A), and survival to discharge (B) according to the transport time interval and prehospital airway management method. BVM, bag-valve-mask; SGA, supraglottic airway; ETI, endotracheal intubation. 
Table 3. Effect of prehospital advanced airway management on survival outcomes according to transport time interval

\begin{tabular}{|c|c|c|c|c|}
\hline & \multicolumn{2}{|c|}{ Good neurologic outcome } & \multicolumn{2}{|c|}{ Survival to discharge } \\
\hline & Crude OR $(95 \% \mathrm{Cl})$ & Adjusted $O R^{a}(95 \% \mathrm{Cl})$ & Crude OR $(95 \% \mathrm{Cl})$ & Adjusted $\left.\mathrm{OR}^{\mathrm{a}}\right)(95 \% \mathrm{Cl}$ \\
\hline \multicolumn{5}{|c|}{$\Pi I \geq 12 \min (n=684)$} \\
\hline BVM & Reference & Reference & Reference & Reference \\
\hline SGA & $1.16(0.65-2.06)$ & $1.37(0.65-2.87)$ & $1.15(0.71-1.87)$ & $1.54(0.85-2.81)$ \\
\hline ETI & $1.03(0.34-3.10)$ & $1.31(0.30-5.81)$ & $1.46(0.63-3.35)$ & $2.48(0.87-7.11)$ \\
\hline \multicolumn{5}{|c|}{$\Pi I<12 \min (n=1,187)$} \\
\hline BVM & Reference & Reference & Reference & Reference \\
\hline SGA & $0.44(0.27-0.72)$ & $0.57(0.31-1.07)$ & $0.54(0.36-0.81)$ & $0.79(0.48-1.31)$ \\
\hline ETI & $0.29(0.07-1.22)$ & $0.63(0.12-3.26)$ & $0.56(0.22-1.44)$ & $1.11(0.38-3.25)$ \\
\hline
\end{tabular}

$\mathrm{OR}$, odds ratio; $\mathrm{Cl}$, confidence interval; $\mathrm{TI}$, transport time interval; $\mathrm{BVM}$, bag-valve-mask; SGA, supraglottic airway; ETI, endotracheal intubation.

${ }^{\text {a) }}$ Adjusted for sex, age, response time interval, scene time interval, location of cardiac arrest occurrence, shockable rhythm, witnessed status, bystander cardiopulmonary resuscitation, prehospital defibrillation, and prehospital epinephrine administration.

Analysis of the association between prehospital airway management and outcomes according to prehospital TTI Among patients in whom the $\pi I$ was $<12$ minutes, those in the SGA and ETI groups had good neurologic outcome rates of 4.4\% and $2.9 \%(P=0.561)$, as well as survival to discharge rates of $7.0 \%$ and $7.3 \%(P=0.950)$, respectively (Fig. 2). After adjustment for potential confounders, neither the use of SGA nor ETI was associated with improved neurologic outcomes in patients with TT/s $\geq 12$ minutes (684 subjects) (OR, $1.37 ; \mathrm{Cl}, 0.65-2.87$ for $\mathrm{SGA} ; \mathrm{OR}$, $1.31 ; \mathrm{Cl}, 0.30-5.81$ for ETI). In patients with Tls $<12$ minutes $(1,197$ subjects), the use of SGA and ETI were also not associated with improved neurologic outcomes $(\mathrm{OR}, 0.57 ; \mathrm{Cl}, 0.31-1.07$ for $\mathrm{SGA} ; \mathrm{OR}, 0.63 ; \mathrm{Cl}, 0.12-3.26$ for $\mathrm{ETI})$ or survival to discharge (OR, $0.79 ; \mathrm{Cl}, 0.48-1.31$ for SGA; OR, $1.11 ; \mathrm{Cl}, 0.38-3.25$ for ETI) compared with BVM ventilation (Table 3).

\section{DISCUSSION}

Our study revealed that the prehospital use of SGA and the performance of ETI were not associated with improved neurologic outcomes or survival to discharge compared to BVM ventilation regardless of transport time.

Conversely, a previous study by Kang et al. ${ }^{12}$ based on a cardiovascular disease surveillance database in South Korea showed significant differences in survival to discharge and good neurologic outcomes between subjects who underwent ETI and those who received BVM; their results were thus inconsistent with ours. The percentages of subjects who underwent ETI and SGA in Kang et al.'s study ${ }^{12}$ study were $3.7 \%$ and $5.0 \%$, respectively, whereas the corresponding percentages in our study were $6.5 \%$ and $51.6 \%$, respectively. Furthermore, the percentage of subjects who underwent bystander CPR in their study (8.3\%) was far lower than in ours (51.4\%). With the gradual increase in the percentage of bystander CPR performed in South Korea, the rates of survival to discharge and good neurologic outcome in our study were relatively higher than those in the previous study. The proportion of EMS personnel who are level 1 EMTs has increased, and a videolaryngoscope to assist EMTs performing ETI has been deployed in ambulances. Furthermore, as the use of I-gel has also increased, the implementation rate of prehospital airway ventilation provided through ETI or the placement of an SGA has also increased. The higher percentage of patients undergoing ETI or SGA placement in our study than in Kang et al.'s study ${ }^{12}$ may have reduced the selection bias.

A recent randomized controlled trial (RCT) on prehospital ventilation support methods based on advanced life support performed in Belgium and France found that BVM was neither inferior nor non-inferior to ETI in terms of survival with favorable 28-day neurological function. ${ }^{3}$ Our present data are consistent with those of the aforementioned RCT, as we found that prehospital advanced airway management is not associated with improved neurologic outcomes and survival to discharge compared to BVM ventilation. Another RCT conducted in England that compared I-gel and ETI found no difference in the rate of favorable neurologic outcomes on the 30th day or at the time of discharge. ${ }^{18}$ However, each country may have differences in the scope of duty and performance level of EMTs. South Korea implements a single-tiered system according to which each ambulance has two or three EMTs. As there are limitations in providing prehospital advanced cardiovascular life support owing to the limited number of EMS personnel, it is difficult to compare data from other countries.

In our study, subjects in the SGA and ETI groups for whom advanced airway management was performed had longer on-scene times and higher rates of prehospital epinephrine administration than did those in the BVM group. Kang et al.'s study ${ }^{12}$ also showed similar results in that subjects for whom prehospital ad- 
vanced airway management was performed had longer on-scene times than did their subjects who underwent BVM. It is presumed that the presence of EMTs who provided advanced care, including advanced airway management and prehospital epinephrine administration, influenced the on-scene time.

We also compared Thls to reflect differences according to region (urban vs. rural) and to medical care accessibility. We hypothesized that prehospital advanced airway management will have advantages over BVM ventilation with respect to neurologic outcomes, and that the benefits of advanced airway management for patients with OHCA will increase with the length of the TI because it can provide effective ventilation and reduce airway complications without interrupting chest compressions. After adjusting for confounding variables, there were no differences in rates of favorable neurologic outcomes among the BVM, SGA, and ETI groups regardless of transport time. A previous study by Wang et al. found that ETI performance was associated with chest compression interruptions, and that the no-flow time was actually extended in situations where CPR was required. ${ }^{19}$ Jarman et al. ${ }^{20}$ reported no differences in CPR interruption time according to the prehospital airway management method, and showed that the failure of the first ETI attempt via direct laryngoscopy actually increased the duration of interrupted chest compressions. Therefore, we must consider the possibility that interrupted chest compressions do not differ according to the airway management method and may actually increase for subjects who undergo advanced airway management; this would explain why advanced management did not affect the neurologic outcomes in real-world situations.

A previous study showed that if the $T \mathrm{TI}$ is expected to be under 14 minutes, it is reasonable to redirect patients experiencing OHCA to percutaneous coronary intervention-capable hospitals. ${ }^{15} \mathrm{Al}-$ though we considered the possibility that the benefits of prehospital advanced airway management during prolonged transfer times may affect the outcomes, we did not identify an association between prehospital advanced airway management and survival outcomes according to TII. Previous studies found that excess hyperventilation after advanced airway management should be avoided because it can cause increased intrathoracic pressure leading to decreased coronary and cerebral perfusion pressure in patients with OHCA. ${ }^{521}$ Kilgannon et al. ${ }^{22}$ also reported that hyperoxia that occurs in patients following resuscitation from cardiac arrest is associated with increased mortality. It is, therefore, possible that these unexpected physiologic effects may offset the potential benefits of proper advanced airway management during transport.

According to a previous study conducted in Japan, a group of subjects in whom advanced airway management was performed within four minutes of CPR initiation showed better neurologic outcomes than did the group in whom advanced airway management was performed after four minutes. ${ }^{23}$ However, conflicting opinions exist regarding the effects of advanced airway management and advanced CPR (including drug use) on site on survival outcomes. ${ }^{24,25}$ Japan implements a single-tiered EMS system similar to that in South Korea; however, emergency life-saving technicians have different ETI certification criteria. ${ }^{23}$ Moreover, the ability to identify the timing of advanced airway management or the cause of its delay in the data registry that we used is limited. Thus, a well-designed follow-up study adapted for the South Korean system is required. In our current study, we were able to identify the association between advanced airway management methods and survival outcomes under the real-life emergency medical system in present-day South Korea. This can help improve future prehospital advanced airway management methods and advanced care directions in multiple-dispatch situations managed by the South Korean emergency medical system.

Our study had a number of limitations that may restrict the generalizability of our results. First, the integrity and validity of the data may be subject to the constraints inherent in multi-institutional observational studies. However, data were collected based on Utstein-style guidelines, and efforts were made to reduce the possibility of potential biases through quality control. Second, the competence levels of prehospital EMTs, regional differences, in-hospital CPR performance levels, and differences in post-cardiac arrest treatments were not reflected in our data; such variables may have influenced our results. Third, although multivariate analyses were performed to adjust for potential confounding variables, the possibility of selection bias still exists. The ETI group comprised the oldest subjects, and differences in the locations of cardiac arrest occurrence were observed; hence, there may have been bias in determining the EMT's attempt and success rates when delivering prehospital advanced airway management to patients with OHCA. Fourth, the actual use of BVM in the field is difficult to identify via hospital records; this may also have contributed to reporting bias. Fifth, the KoCARC registry had insufficient information on the EMTs' level of expertise, patients' initial mental status upon admission to the emergency department, number of advanced airway management attempts, time of intervention, and type of SGA. Therefore, we could not investigate these parameters even though they may influence survival outcomes.

In conclusion, our study revealed no differences in the rates of favorable neurological outcomes between patients with OHCA who were managed using $B V I, E T I$, or SGA, regardless of transport time. 


\section{CONFLICT OF INTEREST}

No potential conflict of interest relevant to this article was reported.

\section{ACKNOWLEDGMENTS}

We would like to acknowledge and thank to investigators from all participating hospitals of KoCARC: Do Kyun Kim (Seoul National University Hospital), Sang Kuk Han, Phil Cho Choi (Kangbuk Samsung Medical Center), Sang 0 Park, Jong Won Kim (Konkuk University Medical Center), Han Sung Choi, Jong Seok Lee (Kyung Hee University Hospital), Sung Hyuk Choi, Young Hoon Yoon (Korea University Guro Hospital), Soo Jin Kim (Korea University Anam Hospital), Min Seob Sim, Gun Tak Lee (Samsung Medical Center), Shin Ahn (Asan Medical Center), Jong Whan Shin (SMG-SNU Boramae Medical Center), Sang Hyun Park, Keun Hong Park (Seoul Medical Center), In Cheol Park, Yoo Seok Park (Yonsei University Severance Hospital), Tae Young Kong (Yonsei University Gangnam Severance Hospital), Kyoung Won Lee, Chu Hyun Kim (Inje University Seoul Paik Hospital), Youngsuk Cho (Hallym University Kangdong Sacred Heart Hospital), Gu Hyun Kang, Yong Soo Jang (Hallym University Kangnam Sacred Heart Hospital), Tai Ho Im, Jae Hoon Oh (Hanyang University Seoul Hospital), Seok Ran Yeom, Sang Kyoon Han (Pusan National University Hospital), Jae Hoon Lee (Dong-A University Hospital), Kyung Woo Lee, Tae Chang Jang (Daegu Catholic University Medical Center), Jae-hyug Woo (Gachon University Gil Medical Center), Woon Jeong Lee, Seon Hee Woo (The Catholic University of Korea Incheon St. Mary's Hospital), Sung Hyun Yun, Tae Jin Cho (Catholic Kwandong University International St. Mary's Hospital), Sun Pyo Kim, Yong Jin Park (Chosun University Hospital), Jin Woong Lee, Wonjoon Jeong (Chungnam National University Hospital), Sung Soo Park, Jae Kwang Lee (Konyang University Hospital), Ryeok Ahn, Wook Jin Choi (UIsan University Hospital), Young Gi Min, Eun Jung Park (Ajou University Hospital), You Hwan Jo, Joong Hee Kim (Seoul National University Bundang Hospital), In Byung Kim, Ki Ok Ahn (Myongji Hospital), Han Jin Cho (Korea University Ansan Hospital), Seung Cheol Lee, Sang Hun Lee (Dongguk University Ilsan Hospital), Young Sik Kim, Young Rock Ha (Bundang Jesaeng Hospital), Jin Sik Park, Myoung Woo Lee (Sejong Hospital), Dai Han Wi (Wonkwang University Sanbon Hospital), Ok Jun Kim, Tae Nyoung Chung (Cha University Bundang Medical Center), Soon Joo Wang, Hang A Park (Hallym University Dongtan Sacred Heart Hospital), Jun Hwi Cho, Chan Woo Park (Kangwon National University Hospital), An Mu Eob, Tae Hun Lee (Hallym University Chuncheon Sacred Heart Hospital), Sang Chul Kim, Hoon Kim (Chungbuk National University
Hospital), Han Joo Choi , Chan Young Koh (Dankook University Hospital), Jung Won Lee, Dong Wook Lee (Soonchunhyang University Cheonan Hospital), Tae Oh Jung, Jae Chol Yoon (Chonbuk National University Hospital), Dai Hai Choi, Jung Tae Choi (Dongguk University Gyeongju Hospital), Jin Hee Jeong, Soo Hoon Lee (Gyeongsang National University Hospital), Ji Ho Ryu, Maeng Real Park (Pusan National University Yangsan Hospital ), Won Kim (Cheju Halla General Hospital), Sung Wook Song, Woo Jung Kim (Jeju National University Hospital), Joon-myoung Kwon, Eui Hyuk Kang (Mediplex Sejong Hospital), Sang Chan Jin, Tae-kwon Kim (Keimyung University Dongsan Medical Center), Hyuk Joong Choi (Hanyang University Guri Hospital), Seong Chun Kim (Gyeongsang National Univerisy Changwon Hospital).

And to steering committee, comprised of following individuals: Sung Oh Hwang (Chair, Wonju Severance Christian Hospital), Sang Do Shin (Chair of Steering Committee, Seoul National University hospital), Hyuk Jun Yang (Advisory Committee, Gachon University Gil hospital), Sung Phil Chung (Data Safety and Management Board, Gangnam Severance Hospital), Sung Woo Lee (Security and Monitoring Board, Korea University Anam hospital), Kyung Jun Song (Secretariat, SMG-SNU Boramae Medical Center), Seung Sik Hwang (Epidemiology and Prevention Research Committee, Seoul National University), Gyu Chong Cho (Community Resuscitation Research Committee, Hallym University Kangdong Sacred Heart Hospital), Sung Woo Moon (Emergency Medical Service Resuscitation Research Committee, Korea University Ansan Hospital), Kyoung Chul Cha (Hospital Resuscitation Research Committee, Wonju Severance Christian Hospital), Won Young Kim (Hypothermia and PostResuscitation Care Research Committee, Asan Medical Center), Sang Hoon Na (Cardiac Care Resuscitation Research Committee, Seoul National University Hospital), Young Ho Kwack (Pediatric Resuscitation Research Committee, Seoul National University hospital).

And lastly to member of Secretariat: Joo Yeong Kim (Korea University Ansan hospital), Jeong Ho Park (Seoul National University hospital), Sun Young Lee (Seoul National University hospital), and Jung Eun Kim (Seoul National University hospital).

\section{REFERENCES}

1. Song KJ, Kim JB, Kim J, et al. Part 2. Adult basic life support: 2015 Korean Guidelines for Cardiopulmonary Resuscitation. Clin Exp Emerg Med 2016;3(Suppl):S10-6.

2. Ocker $H$, Wenzel V, Schmucker P, Dorges V. Effectiveness of various airway management techniques in a bench model simulating a cardiac arrest patient. J Emerg Med 2001;20:7-12.

3. Jabre $P$, Penaloza $A$, Pinero $D$, et al. Effect of bag-mask venti- 
lation vs endotracheal intubation during cardiopulmonary resuscitation on neurological outcome after out-of-hospital cardiorespiratory arrest: a randomized clinical trial. JAMA 2018;319:779-87.

4. Newell C, Grier S, Soar J. Airway and ventilation management during cardiopulmonary resuscitation and after successful resuscitation. Crit Care 2018;22:190.

5. Lee MJ, Rho TH, Kim H, et al. Part 3. Advanced cardiac life support: 2015 Korean Guidelines for Cardiopulmonary Resuscitation. Clin Exp Emerg Med 2016;3(Suppl):S17-26.

6. Yeung J, Chilwan M, Field R, Davies R, Gao F, Perkins GD. The impact of airway management on quality of cardiopulmonary resuscitation: an observational study in patients during cardiac arrest. Resuscitation 2014;85:898-904.

7. Kim YY, Kang GH, Kim WH, et al. Comparison of blind intubation through supraglottic devices and direct laryngoscopy by novices: a simulation manikin study. Clin Exp Emerg Med 2016;3:75-80.

8. Schalk $R$, Meininger $D$, Ruesseler $M$, et al. Emergency airway management in trauma patients using laryngeal tube suction. Prehosp Emerg Care 2011;15:347-50.

9. Jokela J, Nurmi J, Genzwuerker HV, Castren M. Laryngeal tube and intubating laryngeal mask insertion in a manikin by firstresponder trainees after a short video-clip demonstration. Prehosp Disaster Med 2009;24:63-6.

10. Hanif MA, Kaji AH, Niemann JT. Advanced airway management does not improve outcome of out-of-hospital cardiac arrest. Acad Emerg Med 2010;17:926-31.

11. Jeong S, Ahn KO, Shin SD. The role of prehospital advanced airway management on outcomes for out-of-hospital cardiac arrest patients: a meta-analysis. Am J Emerg Med 2016;34: 2101-6.

12. Kang K, Kim T, Ro YS, Kim YJ, Song KJ, Shin SD. Prehospital endotracheal intubation and survival after out-of-hospital cardiac arrest: results from the Korean nationwide registry. Am J Emerg Med 2016;34:128-32.

13. Spaite DW, Bobrow BJ, Vadeboncoeur TF, et al. The impact of prehospital transport interval on survival in out-of-hospital cardiac arrest: implications for regionalization of post-resuscitation care. Resuscitation 2008;79:61-6.
14. Kajino $K$, Iwami T, Daya $M$, et al. Impact of transport to critical care medical centers on outcomes after out-of-hospital cardiac arrest. Resuscitation 2010;81:549-54.

15. Cournoyer A, Notebaert $E$, de Montigny L, et al. Impact of the direct transfer to percutaneous coronary intervention-capable hospitals on survival to hospital discharge for patients with out-of-hospital cardiac arrest. Resuscitation 2018;125:28-33.

16. Geri G, Gilgan J, Wu W, et al. Does transport time of out-ofhospital cardiac arrest patients matter? A systematic review and meta-analysis. Resuscitation 2017;115:96-101.

17. Kim JY, Hwang SO, Shin SD, et al. Korean Cardiac Arrest Research Consortium (KoCARC): rationale, development, and implementation. Clin Exp Emerg Med 2018;5:165-76.

18. Benger JR, Kirby K, Black $S$, et al. Effect of a strategy of a supraglottic airway device vs tracheal intubation during out-ofhospital cardiac arrest on functional outcome: the AIRWAYS-2 randomized clinical trial. JAMA 2018;320:779-91.

19. Wang HE, Simeone SJ, Weaver MD, Callaway CW. Interruptions in cardiopulmonary resuscitation from paramedic endotracheal intubation. Ann Emerg Med 2009;54:645-52.

20. Jarman AF, Hopkins CL, Hansen JN, Brown JR, Burk C, Youngquist ST. Advanced airway type and its association with chest compression interruptions during out-of-hospital cardiac arrest resuscitation attempts. Prehosp Emerg Care 2017;21:628-35.

21. Pitts $S$, Kellermann AL. Hyperventilation during cardiac arrest. Lancet 2004;364:313-5.

22. Kilgannon JH, Jones $\mathrm{AE}$, Shapiro $\mathrm{NI}$, et al. Association between arterial hyperoxia following resuscitation from cardiac arrest and in-hospital mortality. JAMA 2010;303:2165-71.

23. Izawa J, Iwami T, Gibo $K$, et al. Timing of advanced airway management by emergency medical services personnel following out-of-hospital cardiac arrest: a population-based cohort study. Resuscitation 2018;128:16-23.

24. Bakalos G, Mamali M, Komninos $C$, et al. Advanced life support versus basic life support in the pre-hospital setting: a meta-analysis. Resuscitation 2011;82:1130-7.

25. Hagihara A, Onozuka D, Nagata T, Hasegawa M. Effects of advanced life support on patients who suffered cardiac arrest outside of hospital and were defibrillated. Am J Emerg Med 2018;36:73-8. 